\title{
Recruitment and expansion of dendritic cells in vivo potentiate the immunogenicity of plasmid DNA vaccines
}

\author{
Shawn M. Sumida, ${ }^{1}$ Paul F. McKay, ${ }^{2}$ Diana M. Truitt, ${ }^{1}$ Michael G. Kishko, ${ }^{1}$ Janelle C. Arthur, ${ }^{1}$ \\ Michael S. Seaman,, ${ }^{1}$ Shawn S. Jackson,, ${ }^{1}$ Darci A. Gorgone, ${ }^{1}$ Michelle A. Lifton, ${ }^{1}$ \\ Norman L. Letvin, ${ }^{1}$ and Dan H. Barouch ${ }^{1}$ \\ 1Division of Viral Pathogenesis, Beth Israel Deaconess Medical Center, Harvard Medical School, Boston, Massachusetts, USA. \\ 2Department of Cellular and Molecular Medicine, St. George's Hospital Medical School, London, United Kingdom.
}

\begin{abstract}
DCs are critical for priming adaptive immune responses to foreign antigens. However, the utility of harnessing these cells in vivo to optimize the immunogenicity of vaccines has not been fully explored. Here we investigate a novel vaccine approach that involves delivering synergistic signals that both recruit and expand DC populations at the site of antigen production. Intramuscular injection of an unadjuvanted HIV-1 envelope (env) DNA vaccine recruited few DCs to the injection site and elicited low-frequency, env-specific immune responses in mice. Coadministration of plasmids encoding the chemokine macrophage inflammatory protein-1 $\alpha(\mathrm{MIP}-1 \alpha)$ and the DC-specific growth factor fms-like tyrosine kinase 3 ligand with the DNA vaccine resulted in the recruitment, expansion, and activation of large numbers of DCs at the site of inoculation. Consistent with these findings, coadministration of these plasmid cytokines also markedly augmented DNA vaccine-elicited cellular and humoral immune responses and increased protective efficacy against challenge with recombinant vaccinia virus. These data suggest that the availability of mature DCs at the site of inoculation is a critical rate-limiting factor for DNA vaccine immunogenicity. Synergistic recruitment and expansion of DCs in vivo may prove a practical strategy for overcoming this limitation and potentiating immune responses to vaccines as well as other immunotherapeutic strategies.
\end{abstract}

\section{Introduction}

DCs are professional antigen-presenting cells that play a central role in priming immune responses to foreign antigens. Following activation by lipopolysaccharide, cytokines, or other stimuli, immature DCs upregulate expression of MHC and costimulatory molecules and develop into mature DCs that prime T lymphocytes with extraordinary efficiency $(1,2)$. This process effectively initiates immune responses against invading pathogens. However, vaccines may be limited by inadequate numbers of DCs at the site of antigen production to prime immune responses with optimal efficiency.

Plasmid DNA vaccines may be particularly dependent on the availability of DCs for initiating immune responses. DNA vaccines have been shown to elicit immune responses to a diversity of antigens $(3,4)$, but their immunogenicity has proven quite limited. High doses of DNA vaccines are typically required to elicit potent immune responses in mice, and the immunogenicity of DNA vaccines in humans has been marginal to date (5-7). Strategies to augment the immunogenicity of DNA vaccines are therefore being actively developed (8-11). However, the mechanism of immune priming and the factors that limit the immunogenicity of DNA vaccines remain poorly characterized.

Following intramuscular injection of a plasmid DNA vaccine in mice, expression of the encoded antigen occurs primarily in transfected myocytes at the site of inoculation (3). Myocytes lack

Nonstandard abbreviations used: env, envelope; Flt3L, fms-like tyrosine kinase 3 ligand; MIP-1 $\alpha$, macrophage inflammatory protein- $1 \alpha$.

Conflict of interest: The authors have declared that no conflict of interest exists.

Citation for this article: J. Clin. Invest. 114:1334-1342 (2004).

doi:10.1172/JCI200422608 expression of MHC class II and costimulatory molecules and thus would not be expected to prime $\mathrm{T}$ lymphocytes directly. Instead, immune priming likely occurs by DCs $(12-15)$ that presumably migrate to the site of DNA inoculation in response to inflammatory or chemotactic signals following vaccination (16-19). These DCs are thought to present antigen by cross-presentation of extracellular antigen or following direct transfection of plasmid DNA $(13,20,21)$. However, DCs are found only in small numbers and typically exhibit functionally immature phenotypes in nonspecific inflammatory infiltrates. Thus, it is likely that presentation of vaccine-derived antigen to the immune system is an inefficient process that is limited by the availability of functionally mature DCs at the site of inoculation.

In this study, we explored the ability of DC-specific chemotactic and growth factors, specifically plasmid macrophage inflammatory protein-1 $\alpha$ (MIP-1 $\alpha$ ) and plasmid fms-like tyrosine kinase 3 ligand (Flt $3 \mathrm{~L}$ ), to mobilize DCs in vivo and to augment the immunogenicity of plasmid DNA vaccines. We have previously shown that the plasmid chemokine MIP- $1 \alpha$ is able to recruit limited numbers of immature DCs to the site of inoculation in mice $(22,23)$. Flt3L is a potent DC-specific growth factor that has been reported to expand and to mature DCs in both mice and humans $(24,25)$. We therefore reasoned that these signals might exert synergistic combined effects. However, the combination of DC-specific chemotactic and growth factors has not, to our knowledge, previously been investigated. We show here that coadministering plasmid MIP- $1 \alpha$ and plasmid Flt3L with DNA vaccines resulted in a dramatic recruitment and expansion of DCs at the site of antigen production and afforded a substantial augmentation of DNA vaccine immunogenicity and protective efficacy. These studies 


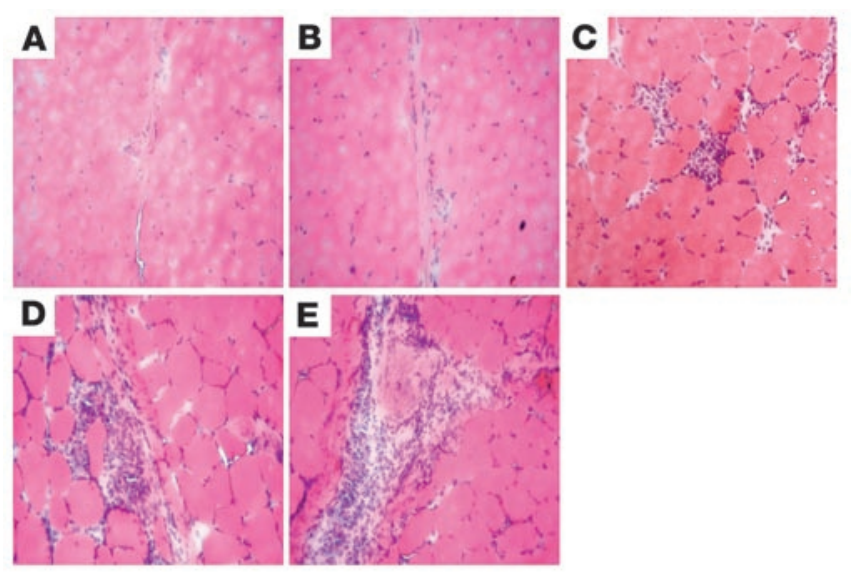

Figure 1

Histopathology of injection sites. BALB/c mice ( $n=4$ per group) were immunized intramuscularly with (A) saline; (B) gp120 DNA vaccine alone; or gp120 DNA vaccine with (C) plasmid Flt3L, (D) plasmid MIP-1 $\alpha$, or (E) both plasmid Flt3L and plasmid MIP-1 $\alpha$. We injected $50 \mu \mathrm{g}$ of each plasmid with sufficient sham plasmid to keep the total DNA dose per mouse constant. Muscle sections ( $5 \mu \mathrm{m}$ thick) were stained with $\mathrm{H} \& \mathrm{E}$ on day 7 following immunization. Magnification, $\times 20$.

demonstrate the potential of harnessing DCs in vivo to enhance vaccine-elicited immune responses.

\section{Results}

Plasmid MIP-1 $\alpha$ and plasmid Flt3L recruit and expand DCs at the site of inoculation. We initiated studies to determine whether codelivering DC-specific chemotactic and growth factors with a plasmid DNA vaccine would lead to increased recruitment and expansion of DCs at the site of vaccine inoculation. We therefore assessed the extent and nature of local cellular inflammatory infiltrates following intramuscular injection of plasmid DNA vaccines with or without plasmids expressing MIP- $1 \alpha$ and Flt3L. Groups of BALB/c mice ( $n=4$ per group) were immunized intramuscularly with sterile saline or $50 \mu \mathrm{g}$ plasmid DNA vaccine expressing HIV-1 IIIB envelope (env) gp120 (16). Certain DNA vaccinated groups were coimmunized with $50 \mu \mathrm{g}$ plasmid Flt3L, $50 \mu \mathrm{g}$ plasmid MIP-1 $\alpha$, or both $50 \mu$ g plasmid MIP- $1 \alpha$ and $50 \mu$ g plasmid Flt3L. Sufficient sham plasmid was included to keep the total dose of DNA per animal constant. The injected muscles were excised on day 7 following immunization, and $5 \mu \mathrm{m}$ serial sections were examined for the presence of local cellular inflammatory infiltrates. As shown in Figure 1, small infiltrates were observed following injection of the DNA vaccine alone. Coimmunization of plasmid Flt $3 \mathrm{~L}$ with the DNA vaccine resulted in slightly larger clusters of inflammatory cells. In contrast, large cellular infiltrates were recruited by plasmid MIP- $1 \alpha$ or the combination of both plasmid cytokines. Quantitation of these inflammatory infiltrates demonstrated that coadministration of both plasmid cytokines resulted in a greater than 10 -fold increase in recruitment of inflammatory cells as compared with the DNA vaccine alone.

We next assessed the nature of these cellular infiltrates by singlecolor immunohistochemistry. As depicted in Figure 2A, none of these sections stained positively for $\mathrm{CD} 3$, indicating that the infiltrates contained few $\mathrm{CD}^{+} \mathrm{T}$ lymphocytes. In contrast, as shown in Figure 2B, we observed substantial differences in CD11b staining among these sections, reflecting variable numbers of CD $11 \mathrm{~b}^{+}$ macrophages or DCs recruited by the various vaccine regimens. Muscle sections from mice immunized with the DNA vaccine alone contained few $\mathrm{CD} 11 \mathrm{~b}^{+}$cells. Plasmid Flt $3 \mathrm{~L}$ recruited limited numbers of additional CD $11 \mathrm{~b}^{+}$cells, indicating small but distinct populations of antigen-presenting cells within heterogeneous cellular infiltrates. Plasmid MIP- $1 \alpha$ and the combination of both plasmid cytokines recruited large infiltrates consisting predominantly of CD $11 \mathrm{~b}^{+}$cells, suggesting that plasmid MIP- $1 \alpha$ exerted a specific chemotactic effect that recruited antigen-presenting cells at the site of inoculation. CD11b is also expressed on NK cells and granulocytes, but we suspect that these cells were not present in large numbers based on subsequently demonstrated staining patterns.

The extent of DC recruitment in these sections using mAbs specific for the DC-specific markers S100 and CD83 is shown in Figure 2, C and D. The DNA vaccine alone recruited few $\mathrm{S}_{100}{ }^{+}$ DCs to the injection site. In contrast, moderate numbers of $S 100^{+}$ DCs were recruited by plasmid Flt3L alone and plasmid MIP- $1 \alpha$ alone. Staining for the DC maturation marker CD83 was low to moderate in these sections, indicating that these DCs had a predominantly immature phenotype. Interestingly, the combination of both plasmid cytokines resulted in massive infiltrates of $\mathrm{S}_{100^{+}}$ DCs that also exhibited high levels of CD83 expression. All sections showed minimal staining for the macrophage-specific marker F4/80 (data not shown).

We further investigated the activation state of the DCs recruited by these vaccine modalities by assessing MHC class II and CD80 expression. As shown in Figure 2, E and F, the cells recruited by plasmid Flt3L alone or plasmid MIP- $1 \alpha$ alone exhibited low to moderate levels of MHC class II and CD80 expression. In contrast, cellular infiltrates recruited by the combination of both plasmid cytokines exhibited high levels of MHC class II and CD80 expression, consistent with a highly activated phenotype. Staining of these sections with an isotype control $\mathrm{mAb}$ was negative (Figure $2 \mathrm{G}$ ).

To analyze these cellular infiltrates in greater detail, we excised muscles from similarly immunized mice on day 7 after injection ( $n=8$ per group), homogenized the tissue, and digested it with collagenase and trypsin. Cell suspensions were then assessed for DCs by staining with $\mathrm{mAbs}$ and 4-color flow cytometric analysis. As shown in Figure 3A, the total cells extracted from muscles injected with plasmid MIP- $1 \alpha$ or both plasmid cytokines was 5 -fold compared to the total cells extracted from muscles injected with the DNA vaccine alone. As depicted in Figure 3B, the number of gated $\mathrm{CD}^{-}{ }^{-} \mathrm{CD} 19^{-}$class $\mathrm{II}^{+} \mathrm{CD} 11 \mathrm{c}^{+}$DCs and activated $\mathrm{CD} 80^{\mathrm{hi}}$ DCs extracted from muscles injected with plasmid MIP-1 $\alpha$ was 5- and 6-fold, respectively, compared with the number of cells extracted from muscles injected with the DNA vaccine alone. Interestingly, the number of DCs and activated CD80hi DCs extracted from muscles injected with both plasmid cytokines was 16- and 27-fold, respectively, compared with the number of cells extracted from muscles injected with the DNA vaccine alone; this is consistent with the results observed in the immunohistochemistry studies. These data demonstrate that plasmid MIP- $1 \alpha$ and plasmid Flt3L exerted synergistic effects that substantially exceeded their additive individual effects.

The large numbers of DCs found in muscles injected with both plasmid cytokines reflected not only a larger number of infiltrating cells, but also a higher percentage of DCs (32\%) in these infiltrates as compared with the infiltrates observed in the other groups (6-8\%) (Figure 3C). Moreover, 77\% of DCs extracted from muscles injected with both plasmid cytokines exhibited high levels of CD80 expres- 
sion as compared with $60 \%$ from muscles injected with plasmid MIP- $1 \alpha, 51 \%$ from muscles injected with plasmid Flt $3 \mathrm{~L}$, and $41 \%$ from muscles injected with the DNA vaccine alone (Figure 3D). These results demonstrate that plasmid MIP- $1 \alpha$ alone was more effective than plasmid Flt3 $\mathrm{L}$ alone in recruiting DCs to the injection site. When these plasmid cytokines were administered together, it is likely that plasmid Flt3L expanded and matured the DC populations recruited by plasmid MIP- $1 \alpha$, thereby resulting in large numbers of mature DCs at the site of inoculation. We observed similar recruitment and activation of DCs when the plasmid cytokines were inoculated without the DNA vaccine (data not shown).

Recruitment of DCs augments

$D N A$ vaccine immunogenicity. We hypothesized that the recruitment, expansion, and maturation of large numbers of DCs at the site of inoculation would likely enhance antigen presentation and immune responses elicited by the DNA vaccine. To explore this possibility, we immunized groups of mice $(n=8$ per group) with sham plasmid; the gp120 DNA vaccine alone; or the gp120 DNA vaccine with plasmid MIP-1 $\alpha$, plasmid Flt3L, or the combination of both plasmid cytokines. We injected $50 \mu \mathrm{g}$ of each plasmid with sufficient sham plasmid to keep the total dose of DNA per animal constant.

Vaccine-elicited CD8 ${ }^{+} \mathrm{T}$ lymphocyte responses specific for the immunodominant $\mathrm{H}-2 \mathrm{D}^{\mathrm{d}}$ restricted P18 epitope (RGPGRAFVTI) (26) were assessed at various time points following immunization by tetramer binding to $\mathrm{CD}^{+} \mathrm{T}$ lymphocytes isolated from peripheral blood $(16,22,27)$. As demonstrated in Figure 4A, following a single injection of the unadjuvanted gp120 DNA vaccine, mice developed peak tetramer ${ }^{+} \mathrm{CD}^{+}$ T lymphocyte responses of $1.3 \%$ on day 10 following immunization. These responses declined

\section{Figure 2}

Immunohistochemistry of injection sites. The muscle sections (5 $\mu \mathrm{m}$ thick) from the vaccinated mice described in Figure 1 were stained with $\mathrm{mAbs}$ specific for murine (A) CD3, (B) CD11b, (C) S100, (D) CD83, (E) MHC class II, (F) CD80, or (G) and isotype control. Magnification, $\times 20$.
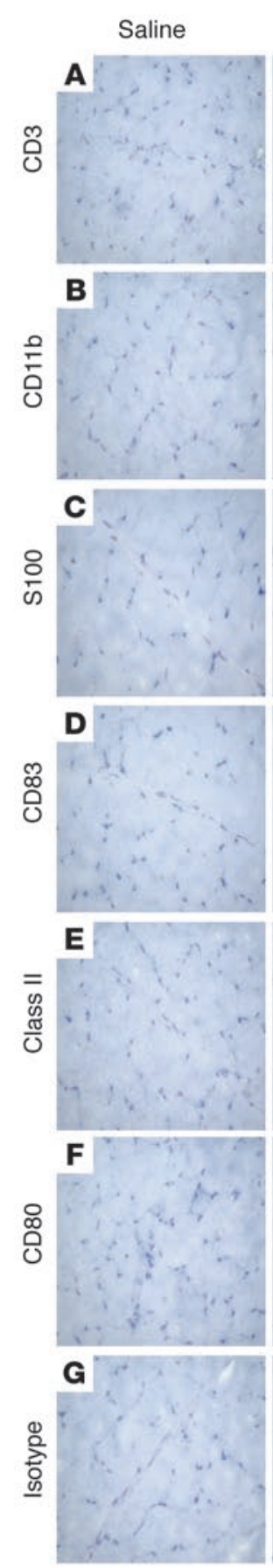

to $0.4 \%$ by day 28 . Addition of plasmid Flt3L had minimal effects on the kinetics or magnitudes of these responses. In contrast, mice that received the DNA vaccine with plasmid MIP-1 $\alpha$ developed higher peak tetramer ${ }^{+} \mathrm{CD}^{+} \mathrm{T}$ lymphocyte responses of $3.4 \%$ on day 10 following immunization. However, this augmentation was transient, and by day 28 , memory tetramer ${ }^{+} \mathrm{CD}^{+} \mathrm{T}$ lymphocyte responses in these mice were indistinguishable from those elicited by the unadjuvanted DNA vaccine. Administering higher doses of plasmid MIP-1 $\alpha$ did not further augment these responses (data not shown). Importantly, mice that received the DNA vaccine with both plasmid cytokines developed peak tetramer ${ }^{+} \mathrm{CD}^{+} \mathrm{T}$
DNA alone
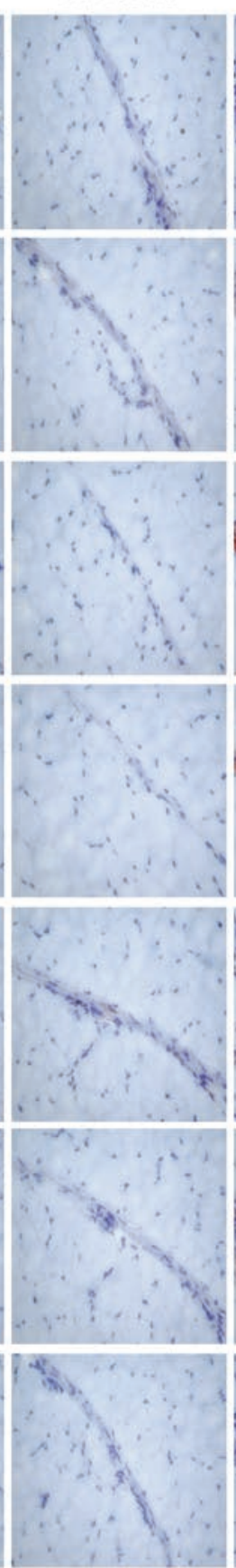

$+\mathrm{Flt} 3 \mathrm{~L}$
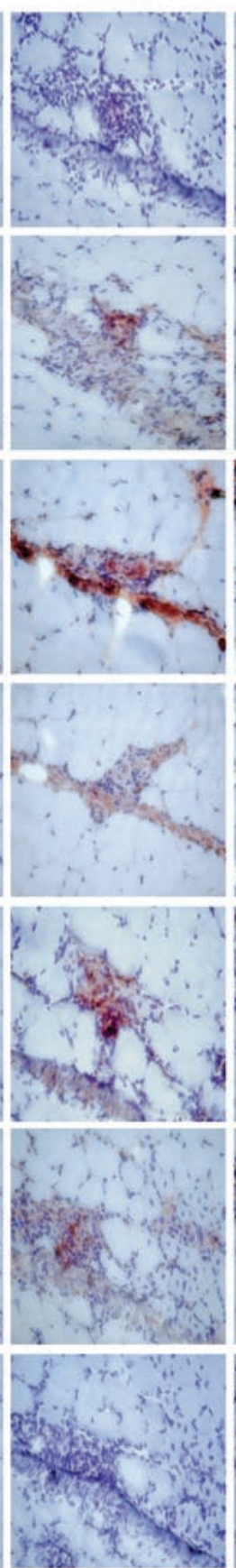

$+\mathrm{MIP}-1 \alpha$
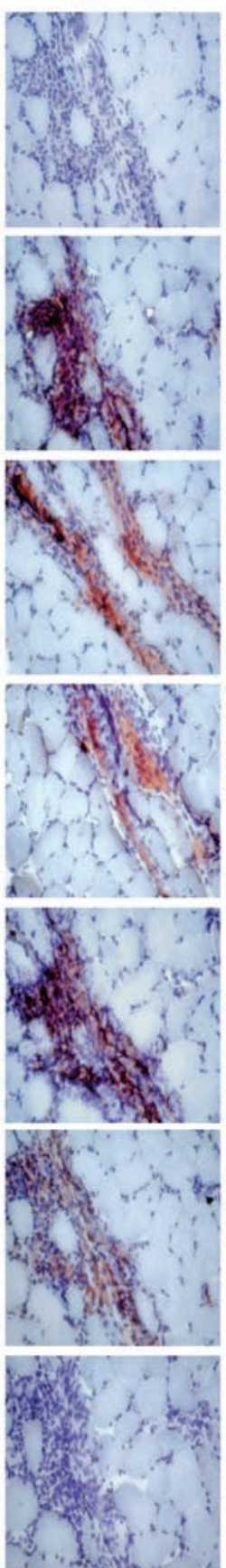

+ MIP $-1 \alpha+$ Flt3L
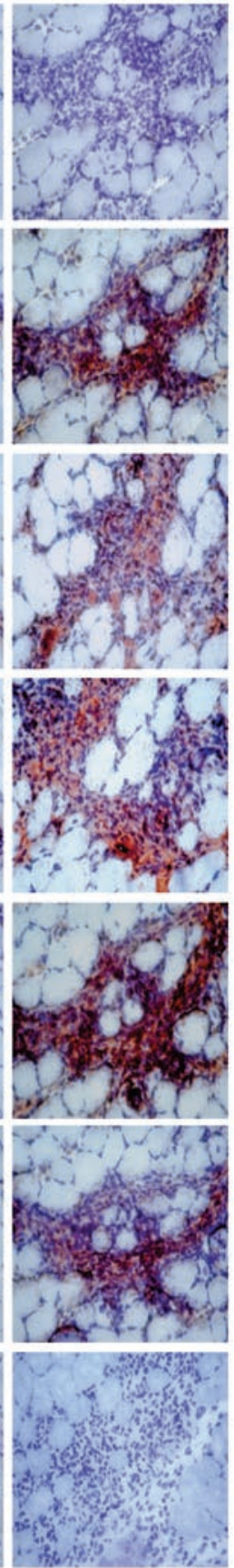

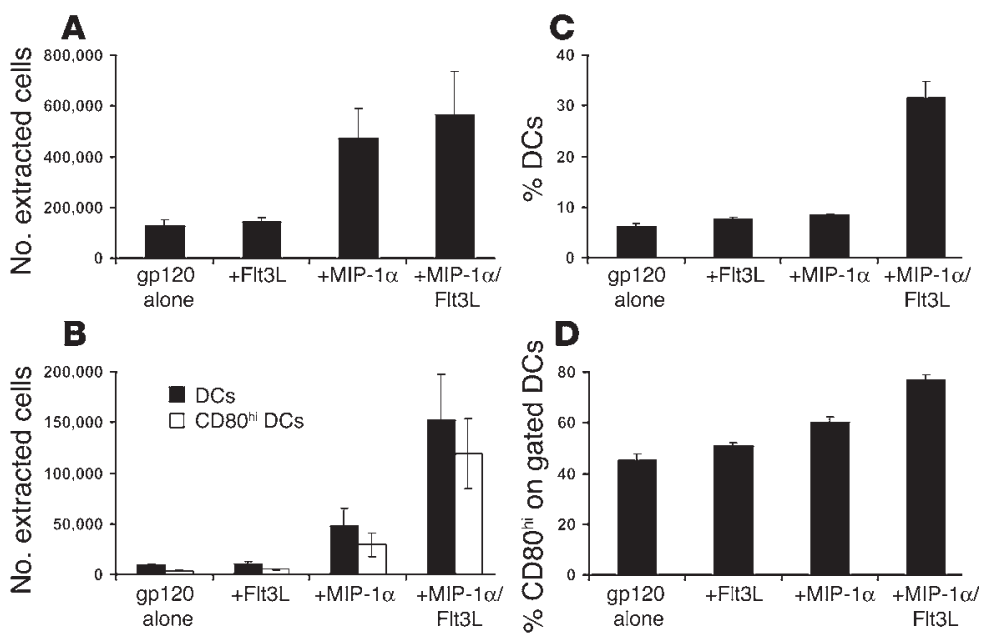

\section{Figure 3}

Analysis of DCs extracted from injected muscles. BALB/C mice were immunized as described in Figure 1. On day 7 following immunization, muscles were excised, homogenized, and digested with collagenase and trypsin ( $n=8$ per group). Cell suspensions were analyzed by 4-color flow cytometry, and DCs were defined as gated CD3-CD19- ${ }^{-}$class $\mathrm{II}^{+}$CD11 $\mathrm{C}^{+}$ cells. (A) Mean total number of extracted cells and (B) mean total number of extracted DCs and CD80 hi DCs per muscle are shown. (C) Percentage of total extracted cells that were DCs and (D) percentage of DCs that were CD80 hi are also shown. In all samples, less than $5 \%$ of the cells were $\mathrm{CD}^{+}$ or CD19+ lymphocytes. lymphocyte responses of $6.1 \%$ on day 10 and maintained memory responses of $1.3 \%$ by day 28 . These responses were significantly higher than those elicited by the unadjuvanted DNA vaccine (5-and 3-fold, respectively; $P<0.001$ comparing groups on day 10 or day 28 using analyses of variance with Bonferroni adjustments to account for multiple comparisons). Tetramer ${ }^{+} \mathrm{CD}^{+} \mathrm{T}$ lymphocyte responses in lymph nodes were comparable with the responses observed in peripheral blood (data not shown). Thus, coadministration of the combination of plasmid MIP- $1 \alpha$ and plasmid Flt3L resulted in a synergistic and durable enhancement of DNA vaccine-elicited $\mathrm{CD}^{+} \mathrm{T}$ lymphocyte responses.

Vaccine-elicited cellular immune responses were also assessed by IFN- $\gamma$ ELISPOT assays using splenocytes harvested on day 28 following immunization and stimulated with a pool of overlapping env peptides or the P18 epitope peptide. As shown in Figure $4 \mathrm{~B}$, vaccine-elicited ELISPOT responses were not detectably augmented by plasmid Flt3L alone or plasmid MIP-1 $\alpha$ alone. Consistent with the tetramer binding assays, mice that received the DNA vaccine with both plasmid cytokines exhibited substantially increased env-specific and P18-specific ELISPOT responses as compared with mice that received the DNA vaccine alone $(P<0.001)$. As demonstrated in Figure 4C, env-specific antibody responses as measured by ELISA were also significantly augmented by these plasmid cytokines $(P<0.01)$. These data show that the recruitment, expansion, and activation of DCs at the site of inoculation using plasmid MIP-1 $\alpha$ and plasmid Flt3L markedly enhanced the magnitude and durability of DNA vaccine-elicited cellular and humoral immune responses.

To explore the generalizability of the adjuvant effects of plasmid MIP- $1 \alpha$ and plasmid Flt3L, we assessed cellular immune responses elicited by the HIV-1 env gp120 DNA vaccine in BALB/c mice and by the SIVmac239 gag DNA vaccine in C57BL/6 mice. As shown in Figure $5 \mathrm{~A}$, coadministration of these plasmid cytokines augmented both pooled peptide and dominant epitope-specific ELISPOT responses in both systems using unfractionated splenocytes, demonstrating that the observed adjuvant effects were neither antigen-specific nor strain-specific. Moreover, as depicted in Figure $5 \mathrm{~B}$, these plasmid cytokines augmented both $\mathrm{CD}^{+}$and $\mathrm{CD} 4^{+}$ $\mathrm{T}$ lymphocyte responses as measured by ELISPOT assays using fractionated splenocyte populations from BALB/c mice.

We next assessed the ability of these primary immune responses to expand following reexposure to antigen. Groups of mice were primed as described in the previous experiment with the gp120 DNA vaccine alone or with plasmid Flt3L, plasmid MIP- $1 \alpha$, or both plasmid cytokines. At week 6 following primary immunization, all groups of vaccinated mice were given booster immunizations of $50 \mu \mathrm{g}$ gp120 DNA vaccine alone to expand the memory $\mathrm{T}$ lymphocyte responses primed by the various vaccine regimens. As shown in Figure 6A, mice primed with the unadjuvanted DNA vaccine developed peak secondary tetramer ${ }^{+}$
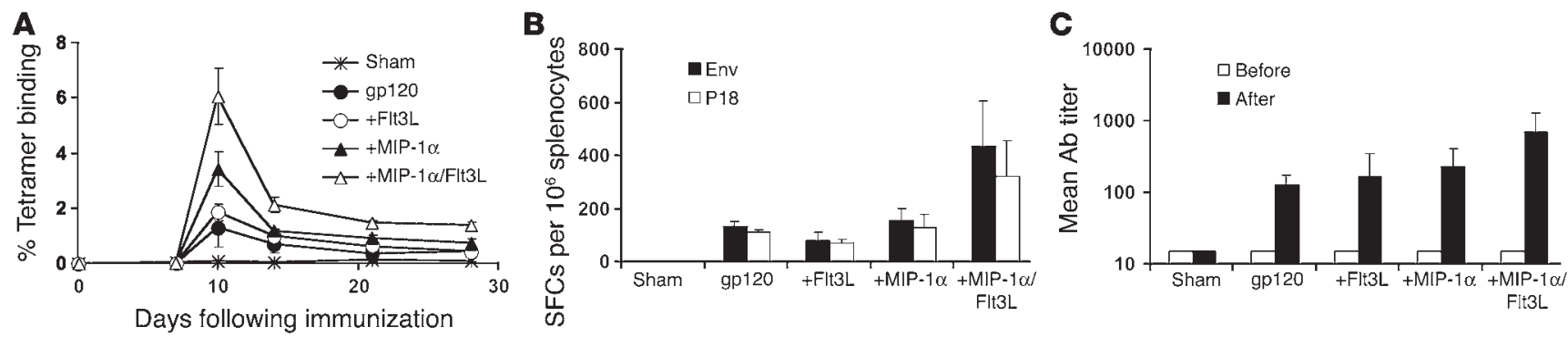

Figure 4

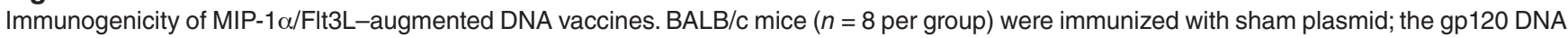
vaccine alone; or the gp120 DNA vaccine with plasmid Flt3L, plasmid MIP-1 $\alpha$, or the combination of both plasmid cytokines. We injected $50 \mu \mathrm{g}$ of each plasmid with sufficient sham plasmid to keep the total DNA dose per mouse constant. Vaccine-elicited immune responses were assessed by (A) Dd/P18 tetramer binding to CD8 ${ }^{+}$T lymphocytes, (B) env-pooled peptide and P18 epitope peptide-specific ELISPOT assays, and (C) gp120-specific ELISAs. The ELISPOT and ELISA assays were performed on day 28 following immunization. SFCs, spot forming cells. 

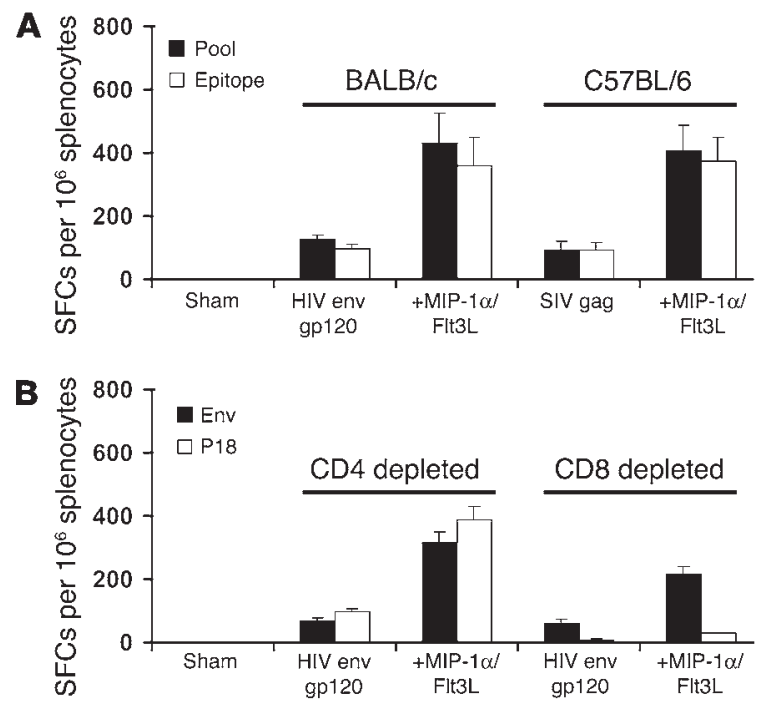

$\mathrm{CD}^{+} \mathrm{T}$ lymphocyte responses of $10.2 \%$ on day 10 following the booster immunization. These responses were not detectably augmented by plasmid Flt3L and were only marginally enhanced by including plasmid MIP- $1 \alpha$ in the priming regimen. Strikingly, mice that were primed with the DNA vaccine with both plasmid MIP- $1 \alpha$ and plasmid Flt $3 \mathrm{~L}$ exhibited peak tetramer ${ }^{+} \mathrm{CD}^{+} \mathrm{T}$ lymphocyte responses of $34.9 \%$ following the booster immunization, demonstrating the substantial potential of memory $\mathrm{CD}^{+} \mathrm{T}$ lymphocytes in these mice to expand rapidly following a booster immunization. As depicted in Figure 6B, substituting a plasmid expressing the costimulatory molecule CD40L in place of plasmid Flt3L abrogated these adjuvant effects. Thus, plasmid MIP- $1 \alpha$ required plasmid Flt3L for synergy, presumably reflecting the ability of Flt3L to expand and mature DCs.

Mechanistic studies of plasmid MIP-1 $\alpha$ and plasmid Flt $3 L$ adjuvanticity. We sought to investigate in greater detail the mechanism of adjuvanticity of plasmid MIP- $1 \alpha$ and plasmid Flt3L. We hypothesized that these plasmid cytokines function by exerting local effects and in particular by recruiting, expanding, and activating DCs at the site of inoculation and antigen production. We first assessed the effects of separating the DNA vaccine and the plasmid cytokines into different muscle groups. Mice were immunized with either 50 $\mu \mathrm{g}$ gp120 DNA vaccine and $50 \mu \mathrm{g}$ of each plasmid cytokine mixed together and delivered equally in both legs, or $50 \mu \mathrm{g}$ gp120 DNA vaccine in the left leg and $50 \mu \mathrm{g}$ of each plasmid cytokine in the right leg. Interestingly, as shown in Figure 7A, anatomic separation of the DNA vaccine and the plasmid cytokines completely abrogated the adjuvanticity of plasmid MIP-1 $\alpha$ and plasmid Flt3L. Thus, these plasmid cytokines exerted predominantly local effects at the site of antigen production.

\section{Figure 6}

Secondary responses following cytokine-augmented DNA vaccine priming and DNA vaccine booster immunization. BALB/c mice $(n=4$ per group) were primed with $50 \mu \mathrm{g}$ gp120 DNA vaccine with or without (A) plasmid MIP-1 $\alpha$ and plasmid Flt3L or (B) plasmid MIP-1 $\alpha$ and plasmid CD40L. At week 6 following immunization, all mice were given booster immunizations of $50 \mu \mathrm{g}$ gp120 DNA vaccine. Vaccine-elicited cellular immune responses were assessed by $\mathrm{D} / \mathrm{P} 18$ tetramer binding to $\mathrm{CD} 8^{+} \mathrm{T}$ lymphocytes following the booster immunization.

\section{Figure 5}

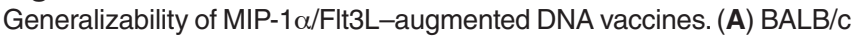
mice or C57BL/6 mice were immunized, respectively, with $50 \mu \mathrm{g}$ HIV env gp120 DNA vaccine or $50 \mu \mathrm{g}$ SIV gag DNA vaccine, each with or without plasmid MIP-1 $\alpha$ and plasmid Flt3L. ELISPOT assays were performed using pooled env peptides and the P18 epitope peptide for the env-vaccinated mice, or pooled gag peptides and the AL11 epitope peptide for the gag-vaccinated mice. (B) ELISPOT assays were performed using splenocytes from env-vaccinated BALB/c mice depleted of $\mathrm{CD}^{+}{ }^{+}$or $\mathrm{CD} 8^{+} \mathrm{T}$ lymphocytes.

We next assessed the effects of disrupting the chemokine gradient established by intramuscular injection of plasmid MIP- $1 \alpha$ by administering high-dose, systemic MIP- $1 \alpha$ protein. Since chemotaxis is dependent on an intact chemokine gradient rather than absolute chemokine concentrations, we hypothesized that disrupting the chemokine gradient would effectively block DC recruitment and abrogate the adjuvanticity of these plasmid cytokines. Mice were immunized with the gp120 DNA vaccine alone or mixed with plasmid MIP-1 $\alpha$ and plasmid Flt3L and also received daily injections of either saline or $1 \mu \mathrm{g}$ recombinant MIP-1 $\alpha$ protein administered i.v. and i.p. We estimate that this dose of recombinant cytokine exceeded the amount expressed by the plasmid by more than 1,000 fold (28). High-dose systemic administration of murine MIP-1 $\alpha$ protein reduced DC recruitment by more than 90\% (data not shown). Accordingly, as shown in Figure 7B, inhibiting DC recruitment also markedly suppressed the adjuvanticity of these plasmid cytokines. These data confirm that the adjuvanticity of plasmid MIP- $1 \alpha$ and plasmid Flt3L required active DC recruitment to the site of inoculation by an intact chemokine gradient.

Intramuscular administration of unadjuvanted DNA vaccines typically requires high doses $(50 \mu \mathrm{g})$ and large injection volumes $(50 \mu \mathrm{l})$ to elicit immune responses in mice. The nonspecific inflammation that occurs at the site of inoculation as a result of these injection parameters likely provides a limited number of antigen-presenting cells that are able to prime low-frequency immune responses. Consistent with prior studies (17), we found that lowering the vaccine dose or the injection volume substantially reduced this inflammation (data not shown) and abrogated vaccine-elicited immune
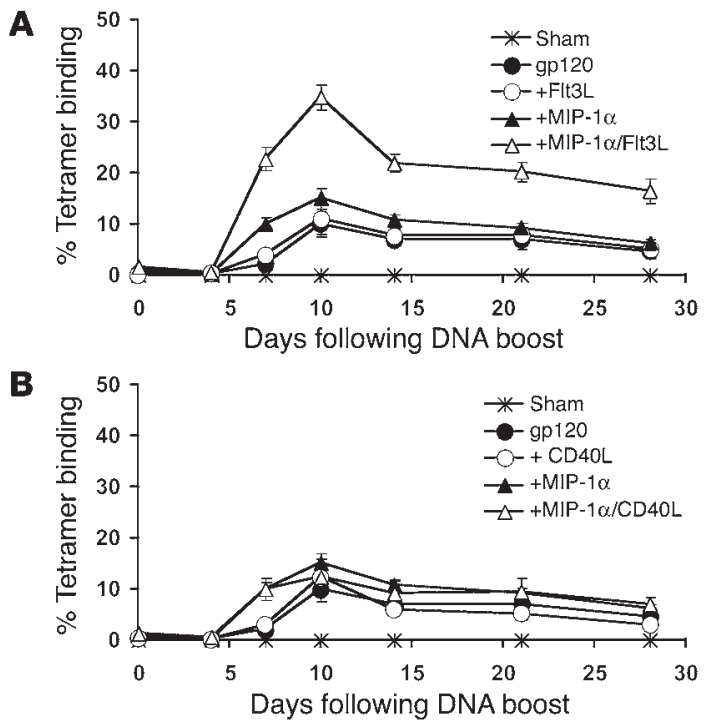

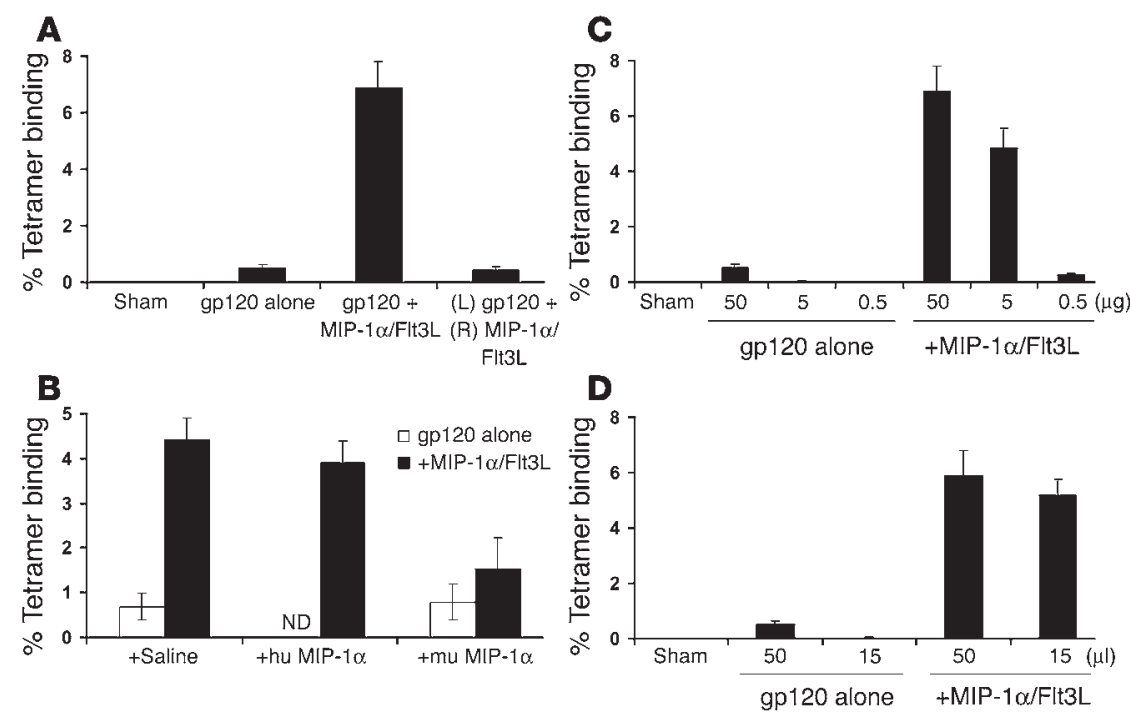

Figure 7

Mechanistic studies for plasmid MIP-1 $\alpha$ and plasmid Flt3L. (A) BALB/c mice were immunized intramuscularly with sham plasmid, gp120 DNA vaccine alone, gp120 DNA vaccine mixed with plasmid MIP-1 $\alpha$ and plasmid Flt3L (delivered equally in both legs), or gp120 DNA vaccine in the left leg (L) and plasmid MIP-1 $\alpha$ and plasmid Flt3L in the right leg (R). (B) Mice were immunized with the gp120 DNA vaccine, with or without plasmid MIP- $1 \alpha$ and plasmid Flt3L, and received daily i.v. and i.p. injections of saline, $1 \mu \mathrm{g}$ human MIP- $1 \alpha$ protein (hu MIP-1 $\alpha$ ), or $1 \mu \mathrm{g}$ murine MIP- $1 \alpha$ protein (mu MIP-1 $\alpha$ ) for 3 days. ND, not done. (C) Mice were immunized with the gp120 DNA vaccine with or without plasmid MIP-1 $\alpha$ and plasmid Flt3L at doses of $50 \mu \mathrm{g}, 5 \mu \mathrm{g}$, or $0.5 \mu \mathrm{g}$ of each plasmid in 50- $\mu$ injection volumes. (D) Mice were immunized with the gp120 DNA vaccine with or without plasmid MIP-1 $\alpha$ and plasmid Flt3L at doses of $50 \mu \mathrm{g}$ of each plasmid in $50-\mu \mathrm{l}$ or $15-\mu \mathrm{l}$ injection volumes. Vaccine-elicited cellular immune responses were assessed by $\mathrm{D}^{\mathrm{d}} / \mathrm{P} 18$ tetramer binding to $\mathrm{CD} 8^{+} \mathrm{T}$ lymphocytes on day 10 following immunization.

responses, depicted in Figure 7, C and D. Interestingly, lowering the dose of the MIP-1 $\alpha /$ Flt3L-augmented DNA vaccine from $50 \mu \mathrm{g}$ to $5 \mu \mathrm{g}$ of each plasmid or lowering the injection volume from $50 \mu \mathrm{l}$ to $15 \mu \mathrm{l}$ had minimal effects on vaccine-elicited tetramer ${ }^{+} \mathrm{CD}^{+} \mathrm{T}$ lymphocyte responses. These data suggest that specific chemotaxis of DCs was substantially more effective than nonspecific inflammation in recruiting DCs to the site of inoculation and in priming immune responses under these limiting conditions.

Recruitment of DCs enhances the protective efficacy of DNA vaccines. To confirm the functional significance of the DNA vaccine-elicited immune responses, we assessed the protective efficacy of these various vaccine regimens against challenge with recombinant vaccinia virus expressing HIV-1 IIIB env. Groups of mice $(n=4$ per group) were immunized with sham plasmid, the gp120 DNA vaccine, or the gp120 DNA vaccine with plasmid MIP-1 $\alpha$ and plasmid Flt3L. We administered $50 \mu \mathrm{g}$ of each plasmid with sufficient sham plasmid to keep the total DNA dose per animal constant. Primary vaccine-elicited tetramer ${ }^{+} \mathrm{CD}^{+} \mathrm{T}$ lymphocyte responses were similar to those shown in Figure 4A, and memory responses were unchanged from week 4 to week 12 following immunization. At week 12, mice were challenged i.p. with $10^{7} \mathrm{PFU}$ recombinant replication-competent vaccinia expressing HIV-1 env IIIB.

Following challenge, we observed anamnestic tetramer ${ }^{+} \mathrm{CD}^{+} \mathrm{T}$ lymphocyte responses in the DNA vaccinated mice as compared with the mice that received the sham plasmid (Figure 8A). Secondary responses were substantially higher in the mice primed with the MIP-1 $\alpha /$ Flt3L-augmented DNA vaccine as compared with mice primed with the unadjuvanted DNA vaccine. Importantly, as shown in Figure 8B, the MIP- $1 \alpha /$ Flt $3 \mathrm{~L}$-augmented DNA vaccine afforded a $2.1 \log$ reduction of vaccinia virus titers in ovaries harvested on day 7 following challenge as compared with sham-vaccinated mice $(P<0.001$ comparing groups using analyses of variance with Bonferroni adjustments). In contrast, the unadjuvanted DNA vaccine afforded only a $0.5 \mathrm{log}$ reduction in vaccinia virus titers as compared with sham-vaccinated mice, reflecting the high stringency of this viral challenge $(P>0.05)$. Thus, the MIP-1 $\alpha /$ Flt3L-augmented DNA vaccine elicited higher pre-challenge primary $\mathrm{CD}^{+} \mathrm{T}$ lymphocyte responses, higher post-challenge anamnestic $\mathrm{CD}^{+} \mathrm{T}$ lymphocyte responses, and improved protective efficacy against a recombinant vaccinia virus challenge as compared with the unadjuvanted DNA vaccine. These studies confirm the functional significance of the enhanced immunogenicity afforded by plasmid MIP-1 $\alpha$ and plasmid Flt3L.

\section{Discussion}

DCs serve a critical role in priming immune responses to foreign antigens. It should therefore be possible to harness these professional antigen-presenting cells in vivo to increase the immunogenicity of vaccines. In this study, we demonstrate that a novel strategy involving both recruitment and expansion of DCs at the site of inoculation using the combination of plasmid MIP- $1 \alpha$ and plasmid Flt $3 \mathrm{~L}$ resulted in a synergistic augmentation of the immunogenicity and protective efficacy of DNA vaccines in mice. Plasmid MIP-1 $\alpha$ alone recruited limited numbers of functionally immature DCs to the site of inoculation and afforded only a transient enhancement of vaccine immunogenicity. Plasmid Flt3L alone was inefficient at mobilizing DCs and exerted no detectable effects on vaccine immunogenicity.

These data are consistent with a model in which plasmid MIP- $1 \alpha$ recruits DCs to the site of inoculation, and plasmid Flt3L triggers the expansion and maturation of these MIP- $1 \alpha$-recruited DCs. However, we cannot exclude the possibility that plasmid Flt3L may also contribute to chemotaxis of DCs, given the pleiotropic effects of this cytokine $(24,25)$. In fact, prior studies have shown that systemic administration of high doses of purified Flt3L protein effectively mobilize DCs in vivo (24). The minimal effects of plasmid Flt3L alone in the present study likely reflected the local and relatively low cytokine expression from plasmid Flt3L. Regardless of the precise mechanism, it is clear that the combination of both plasmid MIP- $1 \alpha$ and plasmid Flt3L was substantially more effective than either plasmid cytokine alone in augmenting DNA vaccine-elicited immune responses.

Our laboratory and others have previously shown that plasmid GM-CSF, plasmid MIP-1 $\alpha$, and other plasmid chemokines can recruit antigen-presenting cells to the site of inoculation and increase vaccine-elicited immune responses in mice $(16,18,22,23$, 29-34). The present study extends these observations by demonstrating that combining a DC-specific chemotactic signal with a 

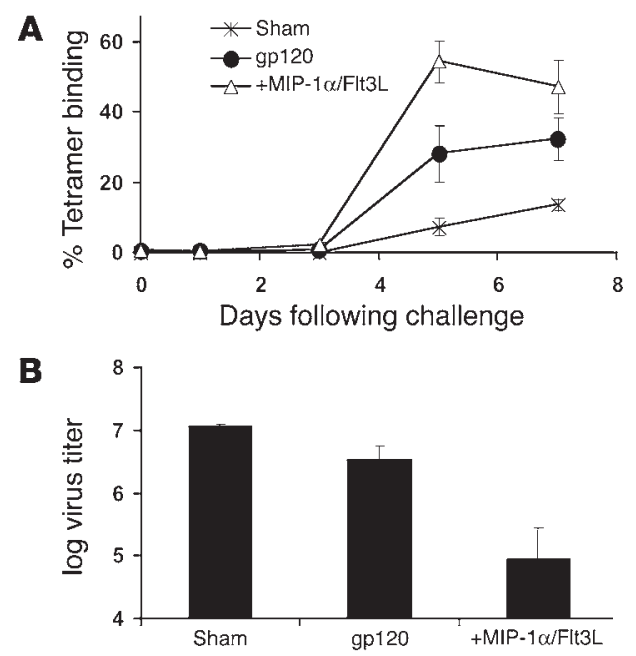

Figure 8

Recombinant vaccinia virus challenge. BALB/c mice ( $n=4$ per group) were immunized intramuscularly with sham plasmid, gp120 DNA vaccine, or gp120 DNA vaccine with plasmid Flt3L and plasmid MIP-1 $\alpha$. At week 12 following immunization, mice were challenged i.p. with $10^{7} \mathrm{PFU}$ recombinant vaccinia virus expressing HIV-1 env IIIB. (A) Anamnestic immune responses were assessed by $\mathrm{D} / \mathrm{P} 18$ tetramer binding to $\mathrm{CD}^{+}$ T lymphocytes following challenge. (B) Vaccinia virus titers (PFU) were assessed in ovaries harvested on day 7 following challenge.

DC-specific expansion and maturation signal results in dramatic and synergistic local effects at the injection site. These studies suggest that both the number and the maturation state of locally available DCs are important in determining the immunogenicity of DNA vaccines. The importance of locally recruited DCs is further underscored by the observation that the adjuvanticity of the plasmid cytokines could be abrogated by ablating the chemokine gradient in vivo or by delivering them at a site that is anatomically distant from the DNA vaccine (Figure 7, A and B). These data demonstrate that efficient DC recruitment required an intact chemokine gradient and that optimal immune priming required functionally mature DCs in close proximity to vaccine-derived antigen.

The capacity of a vaccine to elicit high-frequency immune responses is critically dependent on the efficient presentation of antigen to $\mathrm{T}$ lymphocytes. This may be particularly important for DNA vaccines, which typically express low amounts of antigen that are largely restricted to the local site of inoculation $(3,12,28)$. It should therefore not be surprising that the recruitment, expansion, and maturation of large numbers of DCs at the site of antigen production markedly enhance the immunogenicity of DNA vaccines. We speculate that DCs acquire antigen at the local injection site and then migrate to draining lymph nodes where they prime naive T lymphocytes (17). This possibility is supported by the observation that $\mathrm{T}$ lymphocytes were not found in large numbers in muscle sections (Figure 2A). However, additional detailed studies are clearly required to determine the precise cell trafficking pathways associated with priming immune responses to DNA vaccines.

Our data show that the availability of mature DCs at the site of inoculation may be a critical rate-limiting factor for eliciting high-frequency immune responses to DNA vaccines. Although the present studies were performed in mice, we hypothesize that the immunogenicity of DNA vaccines and perhaps subunit vaccines may similarly be limited by the lack of mature DCs at the site of inoculation in humans. Clinical trials of DNA vaccines utilize substantially lower relative vaccine doses and injection volumes than typical studies in mice after adjusting for body weight. Thus, it is likely that DNA vaccination in humans results in insufficient local inflammation and recruitment of antigen-presenting cells to prime high-frequency immune responses. Coadministration of DC-specific cytokines may prove particularly useful in this setting, since these molecules afforded potent adjuvant effects in mice immunized with limited vaccine doses and injection volumes. Although the practical utility of this strategy will require data from clinical trials, we predict that this strategy of harnessing DCs in vivo will prove effective for both vaccines and other immunotherapeutic interventions.

\section{Methods}

Plasmids, mice, and immunizations. BALB/c or C57BL/6 mice (6-8 weeks old) were purchased from Charles River Laboratories. Mice were injected intramuscularly with the empty VRC-2000 plasmid (Vaccine Research Center, $\mathrm{NIH}$ ) or the same plasmid expressing HIV-1 IIIB env gp120, SIVmac239 gag, murine MIP-1 $\alpha$, murine full-length Flt3L, or murine CD40L. The VRC-2000 plasmid includes a cytomegalovirus (CMV) promoter and enhancer element, a bovine growth hormone (BGH) polyadenylation signal, and a kanamycin resistance gene. Cytokine expression from the various plasmid cytokines was assessed by transient transfection of $1 \mu \mathrm{g}$ DNA in $10^{6} 293 \mathrm{~T}$ cells followed by ELISA analysis of culture supernatants after 48 hours essentially as described (28). Expression from all the plasmid cytokines was efficient and comparable $(50-100 \mathrm{ng} / \mathrm{ml})$. Mice received a single inoculation of $50 \mu \mathrm{g}$ DNA in a $50 \mu \mathrm{l}$ injection volume in the quadriceps muscles unless otherwise noted. All studies with mice were approved by the Institutional Animal Care and Use Committee (IACUC) of Beth Israel Deaconess Medical Center.

Histopathology. Injected murine quadriceps muscles were excised 7 days after immunization and frozen immediately in OCT medium in a dry ice and methanol bath. Frozen muscles were cut into approximately 1,000 serial sections of $5 \mu \mathrm{m}$ thickness, air dried, and fixed for 10 minutes in $100 \%$ acetone. Fixed sections were stained with H\&E before dehydration, mounting, and examination for the presence and extent of cellular inflammatory infiltrates.

Immunohistochemistry. Acetone-fixed 5- $\mu \mathrm{m}$ sections were first treated with $0.5 \%$ hydrogen peroxide in PBS for 15 minutes to quench endogenous peroxidase. The sections were then washed with PBS, and free biotin was blocked using the avidin/biotin blocking system (Vector Laboratories). Sections were then incubated with the primary antibodies at room temperature for 1 hour. Monoclonal antibodies were labeled with biotin and were specific for CD3 (145-2C11; BD Biosciences - Pharmingen), CD11b (M1/70; Insight Biotechnology), S100 (4C4.9; Kamiya Biomedical Co.), CD83 (Michel17; Biocarta US), F4/80 (A3-1; Serotec), MHC class II (M5/114.15.2; Insight Biotechnology), and CD80 (16-10A1; Insight Biotechnology). Isotype control antibodies were also utilized (BD Biosciences - Pharmingen). After incubation, the slides were washed 3 times with PBS and then developed with the Vectastain Elite ABC kit and Vector DAB substrate (Vector Laboratories). After substrate development, the sections were washed in water and counterstained with hematoxylin. Slides were rinsed in water, and coverslips were mounted using histomount (Dako).

Extraction and analysis of infiltrating DCs. Quadriceps muscles from immunized mice were harvested, minced, and incubated with digestion buffer (Hanks' Balanced Salt Solution [Gibco], supplemented with $0.5 \%$ collagenase, $0.2 \% \mathrm{BSA}$, and $0.025 \%$ trypsin) for 30 minutes at $37^{\circ} \mathrm{C}$ with vortexing every 5 minutes to facilitate tissue dissociation. Cell suspen- 
sions were filtered, washed twice with PBS containing $2 \%$ FBS, and stained with FITC-labeled MHC class II (2G9; BD Biosciences - Pharmingen), phycoerythrin-labeled CD11c (HL3; BD Biosciences - Pharmingen), PerCP-Cy5.5-labeled CD3 (145-2C11; BD Biosciences - Pharmingen), PerCP-Cy5.5-labeled CD19 (1D3; BD Biosciences - Pharmingen), and APC-labeled CD80 (16-10A1; eBioscience). Cell suspensions were then washed in PBS containing 2\% FBS and fixed in $0.5 \mathrm{ml}$ PBS containing $1.5 \%$ paraformaldehyde. Samples were analyzed by 4-color flow cytometry on a FACSCalibur (BD Biosciences - Pharmingen). Gated CD3-CD19- class $\mathrm{II}^{+} \mathrm{CD} 11 \mathrm{c}^{+} \mathrm{DCs}$ were quantitated and assessed for CD80 expression.

Tetramer staining. Tetrameric H-2Dd complexes folded around the HIV-1 IIIB V3 loop optimal P18 epitope peptide (P18-I10; RGPGRAFVTI) were prepared and utilized to stain $\mathrm{P} 18$-specific $\mathrm{CD}^{+} \mathrm{T}$ lymphocytes isolated from peripheral blood essentially as described $(16,26,27)$. Mouse blood was collected in RPMI 1640 containing $40 \mathrm{U} / \mathrm{ml}$ heparin. Following lysis of the RBCs, $0.1 \mu \mathrm{g}$ of PE-labeled D $\mathrm{d} / \mathrm{P} 18$ tetramer in conjunction with APClabeled anti-CD8 $\alpha \mathrm{mAb}$ (Ly-2; Caltag) was utilized to stain P18-specific $\mathrm{CD}^{+} \mathrm{T}$ lymphocytes. The cells were washed in PBS containing $2 \% \mathrm{FBS}$ and fixed in $0.5 \mathrm{ml}$ PBS containing $1.5 \%$ paraformaldehyde. Samples were analyzed by 2-color flow cytometry on a FACSCalibur (BD Biosciences Pharmingen). Gated $\mathrm{CD}^{+} \mathrm{T}$ lymphocytes were examined for staining with the $\mathrm{D}^{\mathrm{d}} / \mathrm{P} 18$ tetramer. $\mathrm{CD}^{+} \mathrm{T}$ lymphocytes from naive mice were utilized as negative controls and exhibited less than $0.1 \%$ tetramer staining.

ELISPOT. ELISPOT assays were utilized to assess IFN- $\gamma$ production by splenocytes from vaccinated mice essentially as described (16). The P18 epitope peptide (RGPGRAFVTI) and a pool of overlapping 15 amino acid peptides derived from HIV-1 env IIIB gp120 (Centralised Facility for AIDS Reagents) were utilized for env-vaccinated mice. The AL11 epitope peptide (AAVKNWMTQTL) and a pool of overlapping peptides derived from SIVmac239 gag (AIDS Research and Reference Reagent Program) were utilized for gag-vaccinated mice. We coated 96-well multiscreen plates (Millipore) overnight with $100 \mu \mathrm{l} /$ well of $10 \mu \mathrm{g} / \mathrm{ml}$ rat anti-mouse IFN- $\gamma$ (BD Biosciences - Pharmingen) in PBS, and then washed them with endotoxin-free Dulbecco's PBS (Invitrogen) containing 0.25\% Tween-20 (Sigma-Aldrich) and blocked them with PBS containing 5\% FBS for 2 hours at $37^{\circ} \mathrm{C}$. The plates were washed 3 times with Dulbecco's PBS containing $0.25 \%$ Tween-20, rinsed with RPMI 1640 containing $10 \%$ FBS, and incubated in triplicate with $5 \times 10^{5}$ splenocytes per well in a $100 \mu \mathrm{l}$ reaction volume containing $2 \mu \mathrm{g} / \mathrm{ml}$ peptide. For studies utilizing the peptide pools, each peptide in the pool was present at $2 \mu \mathrm{g} / \mathrm{ml}$. Following an 18-hour incubation, the plates were washed 9 times with Dulbecco's PBS containing $0.25 \%$ Tween- 20 and once with distilled water. The plates were then incubated for 2 hours with $75 \mu \mathrm{l} /$ well of $5 \mu \mathrm{g} / \mathrm{ml}$ biotinylated rat anti-mouse IFN- $\gamma$ (BD Biosciences - Pharmingen), washed 6 times with Coulter Wash (Coulter Corp.), and incubated for 2 hours with a 1:500 dilution of streptavidin-AP (Southern Biotechnology Associates). Following 5 washes with Coulter Wash and 1 with PBS, the plates were developed with nitro blue tetrazolium 5-bromo-4-chloro-3-indolyl-phosphate chromogen (NBT/BCIP chromogen; Pierce Biotechnology), stopped by washing with tap water, air dried, and read using an ELISPOT reader (Hitech Instruments). For depletion studies, splenocytes were incubated with magnetic microbeads coated with anti-CD4 (L3T4) or anti-CD8 (Ly-2) mAbs (Miltenyi Biotec) and separated using MiniMACS columns prior to performing the ELISPOT assay. Cell depletions were more than $95 \%$ efficient.

ELISA. Serum anti-gp120 antibody titers from immunized mice were measured by a direct ELISA (16). We coated 96-well plates overnight with $100 \mu \mathrm{l} /$ well of $1 \mu \mathrm{g} / \mathrm{ml}$ recombinant HIV-1 env IIIB gp120 (Intracel) in PBS, and then blocked them for 2 hours with PBS containing 2\% BSA and $0.05 \%$ Tween-20. Sera were then added in serial dilutions and incubated for 1 hour. The plates were washed 3 times with PBS containing 0.05\% Tween-20 and incubated for 1 hour with a 1:2000 dilution of a peroxidase-conjugated affinity-purified rabbit anti-mouse IgG secondary antibody (Jackson Laboratories) to detect total gp120-specific IgG. The plates were then washed 3 times, developed with tetramethylbenzidine (TMB; Kirkegaard \& Perry Laboratories), stopped with $1 \% \mathrm{HCl}$, and analyzed at $450 \mathrm{~nm}$ with a Dynatech MR5000 ELISA plate reader (Dynatech).

Vaccinia virus challenge. Vaccinated mice were challenged i.p. with $10^{7} \mathrm{PFU}$ replication-competent vaccinia-HIV-1 env IIIB gp160 (BH-10; Therion Biologics) in $100 \mu \mathrm{l}$ sterile PBS. On day 7 following challenge, ovaries were harvested and homogenized by 3 freeze-thaw cycles and vigorous vortexing. The homogenate was trypsinized for 30 minutes at $37^{\circ} \mathrm{C}$. COS-7 cells were plated in 6-well plates at a density of $5 \times 10^{5}$ cells per well and incubated overnight. Cell monolayers were then infected with log dilutions of the homogenate in media. After 2 days, vaccinia virus plaques were visualized by staining with $0.1 \%$ crystal violet and $20 \%$ ethanol.

Statistical analyses. Immunologic data are presented as means with standard errors. Statistical analyses were performed with GraphPad Prism version 4.01 (GraphPad Software Inc.). Comparisons of mean responses among groups of mice were performed by ANOVA with Bonferroni adjustments to account for multiple comparisons. $P$ values of less than 0.05 were considered significant.

\section{Acknowledgments}

We thank Raphael Dolin, Ken Williams, Sampa Santra, Fred Peyerl, Joern Schmitz, Marcelo Kuroda, Gary Nabel, and Dennis Panicali for generous advice, assistance, and reagents. The HIV-1 IIIB env overlapping peptides were obtained from the UK Centralised Facility for AIDS Reagents, and the SIV gag peptides were obtained from the NIH AIDS Research and Reference Reagent Program. We acknowledge support from NIH grants AI-51223 and AI-58727 (to D.H. Barouch) and the Harvard Medical School PASTEUR Program (to S.M. Sumida). D.H. Barouch is also a recipient of a Doris Duke Clinical Scientist Development Award.

Received for publication July 1, 2004, and accepted in revised form August 10, 2004.

Address correspondence to: Dan H. Barouch, Research East Room 113, Division of Viral Pathogenesis, Beth Israel Deaconess Medical Center, 330 Brookline Avenue, Boston, Massachusetts 02215, USA. Phone: (617) 667-4434; Fax: (617) 667-8210; E-mail: dbarouch@bidmc.harvard.edu.
1. Banchereau, J., and Steinman, R.M. 1998. Dendritic cells and the control of immunity. Nature. 392:245-252.

2. Mellman, I., and Steinman, R.M. 2001. Dendritic cells: specialized and regulated antigen processing machines. Cell. 106:255-258.

3. Wolff, J.A., et al. 1990. Direct gene transfer into mouse muscle in vivo. Science. 247:1465-1468.

4. Donnelly, J.J., Ulmer, J.B., Shiver, J.W., and Liu, M.A. 1997. DNA vaccines. Annu. Rev. Immunol.
15:617-648.

5. Calarota, S., et al. 1998. Cellular cytotoxic response induced by DNA vaccination in HIV-1-infected patients. Lancet. 351:1320-1325.

6. MacGregor, R.R., et al. 1998. First human trial of a DNA-based vaccine for treatment of human immunodeficiency virus type 1 infection: safety and host response. J. Infect. Dis. 178:92-100.

7. Wang, R., et al. 1998. Induction of antigen-specific cytotoxic $\mathrm{T}$ lymphocytes in humans by a malaria
DNA vaccine. Science. 282:476-480.

8. Barouch, D.H., et al. 2000. Control of viremia and prevention of clinical AIDS in rhesus monkeys by cytokine-augmented DNA vaccination. Science. 290:486-492.

9. Xiang, Z., and Ertl, H.C. 1995. Manipulation of the immune response to a plasmid-encoded viral antigen by coinoculation with plasmids expressing cytokines. Immunity. 2:129-135.

10. Weiss, W.R., et al. 1998. A plasmid encoding murine 
granulocyte-macrophage colony-stimulating factor increases protection conferred by a malaria DNA vaccine. J. Immunol. 161:2325-2332.

11. Kim, J.J., et al. 1998. Modulation of amplitude and direction of in vivo immune responses by coadministration of cytokine gene expression cassettes with DNA immunogens. Eur. J. Immunol. 28:1089-1103

12. Casares, S., Inaba, K., Brumeanu, T.D., Steinman, R.M., and Bona, C.A. 1997. Antigen presentation by dendritic cells after immunization with DNA encoding a major histocompatibility complex class II-restricted viral epitope. J. Exp. Med. 186:1481-1486.

13. Fu, T.M., et al. 1997. Priming of cytotoxic T lymphocytes by DNA vaccines: Requirement for profession$\mathrm{al}$ antigen presenting cells and evidence for antigen transfer from myocytes. Mol. Med. 3:362-371.

14. Iwasaki, A., et al. 1997. The dominant role of bone marrow-derived cells in CTL induction following plasmid DNA immunization at different sites. J. Immunol. 159:11-14.

15. Torres, C.A.T., Iwasaki, A., Barber, B.H., and Robinson, H.L. 1997. Differential dependence on target site tissue for gene gun and intramuscular DNA immunizations. J. Immunol. 158:4529-4532.

16. Barouch, D.H., et al. 2002. Potent CD4 ${ }^{+} \mathrm{T}$ cell responses elicited by a bicistronic HIV-1 DNA vaccine expressing gp120 and GM-CSF. J. Immunol. 168:562-568.

17. Dupuis, M., et al. 2000. Distribution of DNA vaccines determines their immunogenicity after intramuscular injection in mice. J. Immunol. 165:2850-2858

18. Haddad, D., et al. 2000. Plasmid vaccine express- ing granulocyte-macrophage colony-stimulating factor attracts infiltrates including immature dendritic cells into injected muscles. J. Immunol. 165:3772-3781.

19. Kusakabe, K.I., et al. 2000. The timing of GM-CSF expression plasmid administration influences the Th1/Th2 response induced by an HIV-1-specific DNA vaccine. J. Immunol. 164:3102-3111.

20. Akbari, O., et al. 1999. DNA vaccination: transfection and activation of dendritic cells as key events for immunity. J. Exp. Med. 189:169-178.

21. Chattergoon, M.A., Robinson, T.M., Boyer, J.D., and Weiner, D.B. 1998. Specific immune induction following DNA-based immunization through in vivo trnsfection and activation of macrophages/antigen-presenting cells. J. Immunol. 160:5707-5718.

22. Barouch, D.H., et al. 2003. Plasmid chemokines and colony-stimulating factors enhance the immunogenicity of DNA priming-viral vector boosting HIV-1 vaccines. J. Virol. 77:8729-8735.

23. McKay, P.F., et al. 2004. Recruitment of different subsets of antigen-presenting cells selectively modulates DNA vaccine-elicited CD4+ and CD8+ T lymphocyte responses. Eur. J. Immunol. 34:1011-1020.

24. Maraskovsky, E., et al. 1996. Dramatic increase in the numbers of functionally mature dendritic cells in Flt3 ligand-treated mice: multiple dendritic cell subpopulations identified. J. Exp. Med. 184:1953-1962.

25. Pulendran, B., et al. 1998. Prevention of peripheral tolerance by a dendritic cell growth factor: flt3 ligand as an adjuvant. J. Exp. Med. 188:2075-2082.

26. Takahashi, H., et al. 1992. Induction of broadly cross-reactive cytotoxic T cells recognizing an HIV1 envelope determinant. Science. 255:333-336.
27. Altman, J.D., et al. 1996. Phenotypic analysis of antigen-specific T lymphocytes. Science. 274:94-96.

28. Barouch, D.H., Truitt, D.M., and Letvin, N.L. 2004. Expression kinetics of the interleukin-2/immunoglobulin (IL-2/Ig) plasmid cytokine adjuvant. Vaccine. 22:3092-3097.

29. Mwangi, W., et al. 2002. DNA-encoded fetal liver tyrosine kinase 3 ligand and granulocyte macrophage-colony-stimulating factor increase dendritic cell recruitment to the inoculation site and enhance antigen-specific CD4+ T cell responses induced by DNA vaccination of outbred animals. J. Immunol. 169:3837-3846.

30. Kim, J.J., et al. 1998. CD8 positive T cells influence antigen-specific immune responses through the expression of chemokines. J. Clin. Invest. 102:1112-1124.

31. Kim, J.J., Yang, J.S., Dentchev, T., Dang, K., and Weiner, D.B. 2000. Chemokine gene adjuvants can modulate immune responses induced by DNA vaccines. J. Interferon Cytokine Res. 20:487-498.

32. Sin, J., Kim, J.J., Pachuk, C., Satishchandran, C., and Weiner, D.B. 2000. DNA vaccines encoding interleukin-8 and RANTES enhance antigen-specific Th1-type CD4(+) T-cell-mediated protective immunity against herpes simplex virus type 2 in vivo. J. Virol. 74:11173-11180.

33. Youssef, S., et al. 2000. C-C chemokine-encoding DNA vaccines enhance breakdown of tolerance to their gene products and treat ongoing adjuvant arthritis. J. Clin. Invest. 106:361-371.

34. Karin, N. 2000. Gene therapy for T cell-mediated autoimmunity: teaching the immune system how to restrain its own harmful activities by targeted DNA vaccines. Isr. Med. Assoc. J. 2:63-68. 